\title{
Quantum critical point in the spin glass-Kondo transition in heavy-fermion systems
}

\author{
Alba Theumann \\ Instituto de Física-UFRGS, Av. Bento Gonçalves 9500, 91501-970 Porto Alegre, RS, Brazil \\ B. Coqblin \\ Laboratoire de Physique des Solides, Université Paris-Sud, 91405 Orsay Cedex, France \\ (Received 28 March 2003; revised manuscript received 26 January 2004; published 21 June 2004)
}

\begin{abstract}
The Kondo-spin glass competition is studied in a theoretical model of a Kondo lattice with an intrasite Kondo-type exchange interaction treated within the mean-field approximation, an intersite quantum Ising exchange interaction with random couplings among localized spins, and an additional transverse field $\Gamma$ in the $x$ direction, which represents a simple quantum mechanism of spin flipping, in order to have a better description of the spin glass state and in particular of the quantum critical point (QCP). Taking here a parametrization $\Gamma=\alpha J_{K}^{2}$ (where $J_{K}$ is the antiferromagnetic Kondo coupling), we obtain two second order transition lines from the spin glass state to the paramagnetic one and then to the Kondo state. For a reasonable set of the different parameters, the two second order transition lines do not intersect and end in two distinct QCPs. The existence of a QCP in the spin glass-Kondo competition allows one to give a better description of the phase diagrams of some cerium and uranium disordered alloys.
\end{abstract}

DOI: 10.1103/PhysRevB.69.214418

PACS number(s): 75.20.Hr, 05.50.+q, 64.60.Cn, 75.30.Mb

\section{INTRODUCTION}

It is well known that there exists a strong competition between the Kondo effect on each site of a Kondo lattice and the magnetic ordering arising from the Ruderman-KittelKasuya-Yosida (RKKY) interaction between magnetic atoms in heavy fermion systems. The Doniach diagram ${ }^{1}$ gives a good description of this competition: the Neel temperature $T_{N}$ is first increasing with an increasing of the antiferromagnetic $s$ - $f$ exchange interaction constant $J_{K}(>0)$, then it is passing through a maximum and finally it tends to zero at the "quantum critical point" (QCP), with a second order transition at zero temperature. Such a decrease of $T_{N}$ down to the QCP has been observed in many cerium compounds, such as $\mathrm{CeAl}_{2}$ (Ref. 2) or $\mathrm{CeRh}_{2} \mathrm{Si}_{2}$ (Ref. 3), under pressure. We also know that the Neel temperature starts from zero at a given pressure and increases rapidly with pressure in ytterbium compounds, such as $\mathrm{YbCu}_{2} \mathrm{Si}_{2}$ (Ref. 4) or $\mathrm{YbNi}_{2} \mathrm{Ge}_{2}$ (Ref. 5 ), in good agreement with the Doniach diagram. Above the QCP, there exists a very strong heavy fermion character, but several possible behaviors, i.e., the classical Fermi liquid one with eventually a reduced Kondo temperature ${ }^{6,7}$ or different non-Fermi-liquid (NFL) ones, have been observed in cerium or ytterbium compounds. ${ }^{8,9}$

On the other hand, the disorder can yield a spin glass (SG) phase in addition to the Kondo (mainly NFL) behavior at low temperatures around the QCP in disordered cerium or uranium alloys. This is the case of the magnetic phase diagram of $\mathrm{CeNi}_{1-x} \mathrm{Cu}_{x}$ alloys that has been extensively studied, ${ }^{10,11}$ while the phase diagram of $\mathrm{Ce}_{2} \mathrm{Au}_{1-x} \mathrm{Co}_{x} \mathrm{Si}_{3}$ alloys presents the sequence of SG-AF-Kondo phases at low temperatures with increasing cobalt concentration. ${ }^{12}$ The three phases, AF, SG, and NFL have been obtained at low temperatures for different concentrations in $\mathrm{UCu}_{5-x} \mathrm{Pd}_{x}$ (Ref. 13) or in $\mathrm{U}_{1-x} \mathrm{La}_{x} \mathrm{Pd}_{2} \mathrm{Al}_{3}$ (Ref. 14) disordered alloys. Thus a SG-Kondo transition has been observed experimentally with increasing concentration in disordered alloys around the QCP.

The purpose of our work here is to present a theoretical model that describes the QCP for the spin glass-Kondo transition in Kondo lattices. The SG-Kondo transition was theoretically studied in a previous publication ${ }^{15}$ and also in the presence of ferromagnetic ordering ${ }^{16}$ or antiferromagnetic ordering, ${ }^{17}$ but the QCP was not described because we lacked a quantum mechanism that favored spin flipping. We present here an improvement of the previous model in order to obtain a good description of the QCP.

More precisely, in previous publications ${ }^{15-17}$ the resultant RKKY interaction was introduced by means of random, infinite range couplings among the $S^{z}$ components of the localized spins as in the Sherrington-Kirkpatrick (SK) model ${ }^{18}$ and by neglecting the spin flip coupling of the transverse components. By using functional integral techniques with a static Ansatz in a replica symmetric theory, we obtained a magnetic phase diagram in the $J_{K}$ vs $T$ plane that showed the three different phases: paramagnetic, spin glass, and Kondo. In spite of its complication, the model failed to describe a second order QCP at $T=0$, because, by disregarding the spin flipping part of the Heisenberg Hamiltonian, we suppressed the tunneling mechanism, and magnetic ordering occurs only along the $z$ axis. In order to introduce a spin flipping mechanism and to avoid the intricacies of the random Heisenberg model, ${ }^{19}$ in the present paper the Heisenberg-like coupling among the three spin components induced by the RKKY interaction is mimicked by a quantum Ising spin glass in a transverse field. It consists of an effective random interaction among the $z$ components, as we considered in Ref. 15, while the spin flipping part is simulated by a uniform transverse field in the $x$ direction.

The infinite range quantum Ising spin glass in a transverse field $\Gamma$ is one of the simplest models that presents a quantum critical point and it is equivalent to the model for a spin glass of quantum rotors. ${ }^{20,21}$ The dynamical properties at zero tem- 
perature are known and the existence of the QCP is well established, ${ }^{22}$ while the phase diagram in the $T$ vs $\Gamma$ plane has been studied by using the Trotter-Suzuki technique, ${ }^{23}$ and more recently ${ }^{24}$ by the use of two fermionic representations of the spin operators in terms of Grassmann fields ${ }^{25}$ that are more suitable to our purposes. As we did previously in Ref. 15 , the Kondo effect will be studied in an approximation that is basically equivalent to the mean field decoupling scheme. ${ }^{6,26}$ The static Ansatz in the study of the spin glass transition is an approximation, similar to mean-field theory, that is appropriate to describe the phase diagram. This is justified because in the model of $M$-components quantum rotors, that is exactly soluble for infinite $M$, the critical line is given by the singularity of the zero-frequency mode. ${ }^{20}$ The same occurs with the quantum Ising spin glass in a transverse field, ${ }^{22}$ where the singularity of the zero-frequency mode determines the critical point. That is the reason why the static Ansatz, that describes only the zero-frequency mode, describes the critical line in the phase diagram, although it would not give correctly the time dependence of the order parameter.

A related Hamiltonian has been considered in Ref. 27 to describe NFL behavior and a QCP in some heavy fermion compounds, although there are essential differences between this work and ours in the present paper and in Ref. 15. In Ref. 27 it is considered a system of isolated Kondo impurities, each one with a separate electron reservoir, and represented by the spin glass model of $M$-components quantum rotors in the limit of large $M$, when the problem is exactly soluble. The Kondo coupling provides the quantum mechanism and the transition line in the phase diagram is determined by the singularity of the zero frequency mode, displaying a QCP at zero temperature. The Kondo effect is described there ${ }^{27}$ by isolated impurities and displays a continuous transition among different scaling regimes, in place of the sharp second order transition in Kondo lattices. 1,6,26

This paper is organized as follows: in Sec. II we introduce the model, in Sec. III we discuss relevant results and we reserve Sec. IV for discussions and conclusion. We refer the reader to Ref. 15 for details in the mathematical calculations.

\section{THE MODEL}

We consider a Kondo lattice system with localized spins $\vec{S}_{i}$ at sites $i=1 \cdots N$, coupled to the electrons of the conduction band via a $s-f$ exchange interaction. It is necessary to introduce explicitly the resultant RKKY interaction by means of a random, infinite range coupling among localized spins like in the Sherrington-Kirkpatrick (SK) model for a spin glass. ${ }^{18}$ To describe the Kondo effect in a mean-field-like theory it is sufficient to keep only the spin-flip terms ${ }^{6,15,26}$ in the exchange Hamiltonian, while the spin glass interaction is represented by the quantum Ising Hamiltonian and the transverse field $\Gamma$ in the $x$ direction. ${ }^{24}$ The transverse field $\Gamma$ represents a simple quantum mechanism of spin flipping and mimics the more complicated transverse part of the Heisenberg Hamiltonian. ${ }^{19}$

The Hamiltonian of the model is

$$
\mathcal{H}=\mathcal{H}_{K}+\mathcal{H}_{S G}
$$

$$
\begin{gathered}
\mathcal{H}_{K}=\sum_{k, s} \epsilon_{k} n_{k s}+\epsilon_{0} \sum_{i, s} n_{i s}^{f}+J_{K} \sum_{i}\left[S_{f i}^{+} s_{d i}^{-}+S_{f i}^{-} s_{d i}^{+}\right] \\
\mathcal{H}_{S G}=-\sum_{i, j} J_{i j} S_{f i}^{z} S_{f j}^{z}-2 \Gamma \sum_{i} S_{f i}^{x}
\end{gathered}
$$

where $J_{K}>0, S^{ \pm}=S^{x} \pm i S^{y}$, and

$$
\begin{aligned}
& \vec{S}_{f i}=\sum_{s, s^{\prime}} f_{i s}^{\dagger} \vec{\sigma}_{s, s^{\prime}} f_{i s^{\prime}}, \\
& \vec{s}_{d i}=\sum_{s, s^{\prime}} d_{i s}^{\dagger} \vec{\sigma}_{s, s^{\prime}} d_{i s^{\prime}},
\end{aligned}
$$

$f_{i s}^{\dagger}, f_{i s}\left(d_{i s}^{\dagger}, d_{i}\right)$ are creation and destruction fermion operators for electrons with $s=\uparrow$ or $\downarrow$ in the localized (conduction) band. We indicate by $\vec{\sigma}$ the Pauli matrices and we have $n_{k \sigma}$ $=d_{k \sigma}^{\dagger} d_{k \sigma}$ the conduction electrons occupation number. The energies $\epsilon_{0}\left(\epsilon_{k}\right)$ are referred to the chemical potentials $\mu_{f}\left(\mu_{d}\right)$, respectively.

The coupling $J_{i j}$ in Eq. (3) is an independent random variable $^{18}$ with a Gaussian distribution of zero mean and variance $\left\langle J_{i j}^{2}\right\rangle=8 J^{2} / N$.

Functional integration techniques have proved to be a suitable approach to describe phase transitions in disordered quantum mechanical many-particle systems. ${ }^{25}$ The static approximation within this formulation consists of neglecting time fluctuations of the order parameter, and when it is combined with the neglect of space fluctuations it leads to the usual Hartree-Fock, mean-field-like approximation. When dealing with the Hamiltonian in Eqs. (1)-(3), we notice that in the limiting case $J_{K}=0$ we obtain a quantum Ising spin glass in a transverse field that has been studied with the static approximation in Ref. 24, while for $J=0$ we recover the mean-field approximation that has been used successfully to describe the Kondo lattice. ${ }^{6,8,26}$ We follow closely the formalism of Ref. 15 to write a Lagrangian formulation ${ }^{25}$ in terms of anticommuting, complex Grassmann variables $\varphi_{i s}(\omega), \psi_{i s}(\omega)$ associated to the conduction and localized electron fields, respectively, together with the spinors

$$
\varphi_{i}(\omega)=\left(\begin{array}{c}
\varphi_{i \uparrow}(\omega) \\
\varphi_{i \downarrow}(\omega)
\end{array}\right), \quad \underline{\psi}_{i}(\omega)=\left(\begin{array}{c}
\psi_{i \uparrow}(\omega) \\
\psi_{i \downarrow}(\omega)
\end{array}\right)
$$

where $\omega=(2 n+1) \pi$ are the Matsubara frequencies. The partition function is now expressed as

$$
Z=\int D\left(\varphi^{\dagger} \varphi\right) D\left(\psi^{\dagger} \psi\right) e^{A}
$$

We now follow the same steps as in Refs. 15-17 and in the static, mean-field-like approximation the action $A$ may be written

$$
A=A_{0}+A_{K}+A_{S G},
$$


with $A_{0}$ being the action for noninteracting electrons in a transverse magnetic field:

$$
\begin{aligned}
A_{0}= & \sum_{\omega} \sum_{i, j} \underline{\psi}_{i}^{\dagger}(\omega)\left[\left(i \omega-\beta \epsilon_{0}+\beta \Gamma \underline{\sigma}_{x}\right) \delta_{i j} \underline{\psi}_{i}(\omega)\right. \\
& \left.+\varphi_{i}^{\dagger}\left(i \omega \delta_{i j}-\beta t_{i j}\right) \underline{\varphi}_{j}(\omega)\right]
\end{aligned}
$$

while the Kondo part of the action is decoupled in the meanfield approximation as in Ref. 15

$$
A_{K}=\frac{\beta J_{K}}{N} \sum_{\sigma}\left[\sum_{i \omega} \psi_{i \sigma}^{\dagger}(\omega) \varphi_{i \sigma}(\omega)\right]\left[\sum_{i, \omega} \varphi_{i-\sigma}^{\dagger} \psi_{i-\sigma}(\omega)\right] .
$$

Here $\beta$ is the inverse absolute temperature and we also have

$$
A_{S G}=\sum_{i, j} J_{i j} S_{f i}^{z} S_{f j}^{z}
$$

where in the static approximation ${ }^{15-17,24}$

$$
S_{f i}^{z}=\frac{1}{2} \sum_{\omega} \psi_{i}^{\dagger}(\omega) \sigma_{-}^{z} \psi_{i}(\omega) .
$$

The Kondo order is described by the complex order parameter

$$
\begin{aligned}
& \lambda_{s}^{\dagger}=\frac{1}{N} \sum_{i, \omega}\left\langle\psi_{i s}^{\dagger}(\omega) \varphi_{i s}(\omega)\right\rangle, \\
& \lambda_{s}=\frac{1}{N} \sum_{i, \omega}\left\langle\varphi_{i s}^{\dagger}(\omega) \psi_{i s}(\omega)\right\rangle
\end{aligned}
$$

that in a mean-field theory ${ }^{6,15,26}$ describes the correlations $\lambda_{s}^{\dagger}=\left\langle f_{i s}^{\dagger} d_{i s}\right\rangle$ and $\lambda_{s}=\left\langle d_{i s}^{\dagger} f_{i s}\right\rangle$. The approximation used in Eq. (12), which is equivalent to the "slave boson" method, is certainly one of the best practical methods used for the Kondo lattice problem. The Kondo temperature for the lattice is determined here by the temperature at which $\lambda$ becomes equal to zero and this approximation gives a fairly reasonable description of the Kondo phase. However, it is unable to give a "mixed" SG-Kondo phase, as it was previously shown in the case of the antiferromagnetic-Kondo transition. ${ }^{28}$ Except for the presence of the transverse field $\Gamma$ in $A_{0}$ in Eq. (8), the calculation follows the same steps as in Ref. 15 and we refer the reader to this paper for details. Reference 15 shows that standard manipulations give for the averaged free energy within a replica symmetric theory:

$$
\beta F=2 \beta J_{K} \lambda^{2}+\frac{1}{2} \beta^{2} J^{2}\left(\bar{\chi}^{2}+2 q \bar{\chi}\right)-\beta \Omega
$$

where

$$
\begin{aligned}
\beta \Omega= & \lim _{n \rightarrow 0} \frac{1}{N n}\left\{\int_{-\infty}^{+\infty} \prod_{j}^{N} D z_{j} \prod_{\alpha}^{n} \int_{-\infty}^{+\infty} \prod_{j} D \xi_{\alpha j}\right. \\
& \left.\times \exp \left[\sum_{\omega} \ln \left|\underline{G}_{i j \alpha}^{-1}(\omega)\right|\right]-1\right\}
\end{aligned}
$$

and $q, \bar{\chi}$, and $\lambda$ must be taken at their saddle-point value. Here $\alpha$ is the replica index and $q$ is the SG order parameter ${ }^{15,18,24}$ while $\chi=\beta \bar{\chi}$ is the static uniform spin susceptibility of the localized $f$ electrons.

$$
\begin{gathered}
q_{\alpha \neq \beta}=q=\lim _{n \rightarrow 0} \frac{1}{n(n-1)} \sum_{\alpha \neq \beta}\left\langle S_{\alpha}^{z} S_{\beta}^{z}\right\rangle, \\
q_{\alpha \alpha}=q+\bar{\chi}=\lim _{n \rightarrow 0 n} \frac{1}{n} \sum_{\alpha}\left\langle S_{\alpha}^{z} S_{\alpha}^{z}\right\rangle .
\end{gathered}
$$

We use the notation $D x=(d x / \sqrt{2 \pi}) e^{-1 / 2 x^{2}}$ and we refer the reader to Ref. 15 for the details of the calculation. The matrix $G_{i j \alpha}(\omega)$ in Eq. (14) is the time Fourier transform of the matrix Green's function $\underline{G}_{i j}(\tau)=i\left\langle T \underline{\psi}_{i}(\tau) \underline{\psi}_{j}^{\dagger}(0)\right\rangle$ for the localized electrons in the presence of random fields $z_{j}$ and $\xi_{\alpha j}$ at every site, and satisfies the equation ${ }^{15}$

$$
\underline{G}_{i j \alpha}^{-1}(\omega)=\left[i \omega-\beta \epsilon_{0}-\underline{\sigma}_{z} h_{j \alpha}+\beta \Gamma \underline{\sigma}_{x}\right] \delta_{i j}-\beta^{2} J_{K}^{2} \lambda^{\dagger} \lambda \gamma_{i j}(\omega) \underline{1}
$$

where

$$
h_{j \alpha}=\sqrt{2 q} \beta J z_{j}+\sqrt{2 \bar{\chi}} \beta J \xi_{\alpha j}
$$

while $\gamma_{i j}(\omega)$ is the time Fourier transform of the conduction electron Green's function $\gamma_{i j}(\tau)=i\left\langle T \varphi_{i s}(\tau) \varphi_{i s}(0)\right\rangle$ and is given by

$$
\gamma_{i j}^{-1}=\left[i \omega-\beta \mu_{c}\right] \delta_{i j}-\beta t_{i j} .
$$

We obtained in Eq. (17) the Green's function for the $f$ electrons in a Kondo lattice, ${ }^{15}$ but now the presence of a random field $h_{j}$ at every site prevents us from diagonalizing by means of a Fourier transformation. In the pure SG limit $J_{K}$ $=0$, the Green's function in Eq. (17) is local and the integrals in Eq. (14) reduce to a one site problem, while in the Kondo limit $J=0$ the random fields vanish and the integrals separate in reciprocal space, giving the known results for a Kondo lattice. We adopt here a decoupling approximation introduced in Ref. 15 that reproduces correctly these two limits, and corresponds to consider independent Kondo lattices, in place of the independent Kondo impurities in Ref. 27. We replace the Green's function $\underline{G}_{i j \alpha}\left(\omega, h_{1 \alpha}, \ldots, h_{j \alpha}, \ldots, h_{N \alpha}\right)$ by the Green's functions $\underline{G}_{\mu \nu}\left(\omega, h_{j \alpha}\right)$ with $j=1, \ldots, N$, of $N$ independent Kondo lattices, each one with a "uniform" field $h_{j \alpha}$ at every site $\mu, \nu$ by means of the approximation

$$
\ln \left|\underline{G}_{i j \alpha}^{-1}\left(\omega, h_{1}, \ldots, h_{N}\right)\right| \approx \frac{1}{N} \sum_{j} \ln \left|\underline{G}_{\mu \nu}^{-1}\left(\omega, h_{j \alpha}\right)\right|
$$

where $\underline{G}_{\mu \nu}\left(\omega, h_{j \alpha}\right)$ is the $f$-electron Green's function for a fictitious Kondo lattice that has a uniform field $h_{j \alpha}$ at every site $\mu, \nu$ and satisfies the equation:

$$
\begin{aligned}
\underline{G}_{\mu \nu}^{-1}\left(\omega, h_{j \alpha}\right)= & {\left[\left(i \omega-\beta \epsilon_{0}\right) \underline{1}-\underline{\sigma}_{z} h_{j \alpha}+\beta \Gamma \underline{\sigma}_{x}\right] \delta_{\mu \nu} } \\
& -\beta^{2} J_{K}^{2} \lambda^{2} \gamma_{\mu \nu},
\end{aligned}
$$

where from Eq. (19) 


$$
\gamma_{\mu \nu}(\omega)=\frac{1}{N} \sum_{k} \frac{1}{i \omega-\beta \epsilon_{k}} e^{i \vec{k} \cdot \vec{R}_{\mu \nu}}
$$

Now Eq. (21) may be easily solved by a Fourier transformation with the result:

$$
\ln \left|\underline{G}_{\mu \nu}^{-1}\left(\omega, h_{j \alpha}\right)\right|=\sum_{\vec{k}} \ln \left|\underline{G}_{\vec{k}}^{-1}\left(\omega, h_{j \alpha}\right)\right|
$$

where

$$
\underline{G}_{\vec{k}}^{-1}\left(\omega, h_{j \alpha}\right)=\left[i \omega-\beta \epsilon_{0}\right] \underline{1}-\underline{\sigma}_{z} h_{j \alpha}+\beta \Gamma \underline{\sigma}_{x}-\beta^{2} J_{K}^{2} \lambda^{2} \frac{1}{i \omega-\beta \epsilon_{k}} .
$$

We may now introduce Eqs. (24) and (20) in Eq. (14), the integrals over the fields separate and we obtain

$$
\beta \Omega=\int_{-\infty}^{+\infty} D z \ln \left\{\int_{-\infty}^{+\infty} D \xi \exp \left[\frac{1}{N} \sum_{\vec{k}} \sum_{s} S_{s}(\vec{k}, H)\right]\right\}
$$

with

$$
\sum_{s} S_{S}(\vec{k}, H)=\sum_{\omega} \ln \left[\underline{G}_{\vec{k}}^{-1}(\omega, h)\right]
$$

and $h$ is given in Eq. (18), with $z$ and $\xi$ in place of $z_{j}$ and $\xi_{j \alpha}$, while

$$
H=\sqrt{h^{2}+(\beta \Gamma)^{2}} .
$$

The sum over the fermion frequencies is performed in the standard way by integrating in the complex plane ${ }^{25}$ with the result:

$$
S_{s}(\vec{k}, H)=\ln \left[\left(1+e^{-\omega_{s^{+}}}\right)\left(1+e^{-\omega_{s^{-}}}\right)\right]
$$

where

$$
\omega_{s^{ \pm}}=\frac{1}{2}\left[\beta \epsilon_{k}+s H\right] \pm\left\{\frac{1}{4}\left(\beta \epsilon_{k}-s H\right)^{2}+\left(\beta J_{K} \lambda\right)^{2}\right\}^{1 / 2} .
$$

We consider $\epsilon_{0}=0$ that corresponds to an average occupation $\left\langle n_{f}\right\rangle=1$, per site. Replacing sums by integrals, in the approximation of a constant density of states for the conduction band electrons, $\rho(\epsilon)=\rho=1 / 2 D$ for $-D<\epsilon<D$, we obtain from Eqs. (26)-(29) the final expression for the free energy in Eq. (13):

$$
\begin{aligned}
\beta F= & 2 \beta J_{K} \lambda^{2}+\frac{1}{2} \beta^{2} J^{2}\left(\bar{\chi}^{2}+2 q \bar{\chi}\right) \\
& -\int_{-\infty}^{+\infty} D z \ln \left\{\int_{-\infty}^{+\infty} D \xi e^{E(H)}\right\}
\end{aligned}
$$

with

$$
E(H)=\frac{1}{\beta D} \int_{-\beta D}^{+\beta D} d x \ln \left\{\cosh \frac{(x+H)}{2}+\cosh (\sqrt{\Delta})\right\},
$$

$$
\Delta=\frac{1}{4}(x-H)^{2}+\left(\beta J_{K} \lambda\right)^{2},
$$

and from Eq. (27) we have

$$
H=\sqrt{(\beta \Gamma)^{2}+(\beta J)^{2}(\sqrt{2 q} z+\sqrt{2 \bar{\chi}} \xi)^{2}} .
$$

\section{RESULTS}

The SG-Kondo transition is described in the present model by the three parameters $J_{K}, J$, and $\Gamma$, which cannot be considered here as completely independent from each other. In fact, the Kondo effect and the RKKY interaction originate from the same intrasite exchange interaction, but the necessity of considering an additional intersite exchange term has been already recognized ${ }^{6,28}$ and a relationship giving the intersite exchange parameter varying as $\left(J_{K}\right)^{2}$ has been introduced in Ref. 6. Since the transverse field is introduced here to mimic the spin flipping part of the Heisenberg Hamiltonian that originates in the RKKY interaction, we assume a similar relationship $\Gamma=\alpha\left(J_{K}\right)^{2}, \alpha \leqslant 1$ to have a better description of the SG-Kondo transition. In particular, the consideration of the previous relationship for $\Gamma$ will be important for the existence of the QCP and the comparison with experiment. The saddle-point equations for $\bar{\chi}, q$, and $\lambda$ are obtained by extremizing $\beta F$ with respect to these order parameters. By making $q=\lambda=0$ in the saddle-point equations we obtain the equations for the second order critical lines $T_{1}\left(J_{K}\right)$ and $T_{2}\left(J_{K}\right)$ that separate the paramagnetic phase from the spin glass and Kondo phases, respectively. They are given by

$$
\bar{\chi}(\beta)=\frac{1}{1+J_{0}} \int_{-\infty}^{+\infty} D \xi \xi^{2} \sinh \left(H_{0}\right) / H_{0}
$$

where

$$
J_{0}=\int_{-\infty}^{+\infty} D \xi \cosh \left(H_{0}\right)
$$

and $H_{0}=H(q=0)$.

Thus we obtain for the spin glass transition temperature $T_{1}\left(J_{K}\right):{ }^{24}$

$$
\bar{\chi}\left(\beta_{1}\right)=\frac{1}{\sqrt{2} \beta_{1} J}
$$

that together with Eq. (34) gives $T_{1}$ and $\bar{\chi}\left(\beta_{1}\right)$, while the Kondo transition temperature $T_{2}\left(J_{K}\right)$ is given by

$$
\begin{aligned}
& 1-\frac{\beta_{2} J_{k}}{4\left(1+J_{0}\right)} \int_{-\infty}^{+\infty} D \xi \cosh \left(H_{0} / 2\right) \\
& \quad \times \frac{1}{\beta_{2} D} \int_{-\beta_{2} D}^{+\beta_{2} D} d x \frac{1}{\cosh (x / 2)} \frac{\sinh \left(\frac{H_{0}-x}{2}\right)}{\frac{H_{0}-x}{2}}=0
\end{aligned}
$$

that must be solved together with Eq. (34).

The numerical solution of the saddle-point equations as a function of $T / J$ and $J_{K} / J$ yields the phase diagram in Fig. 1, 


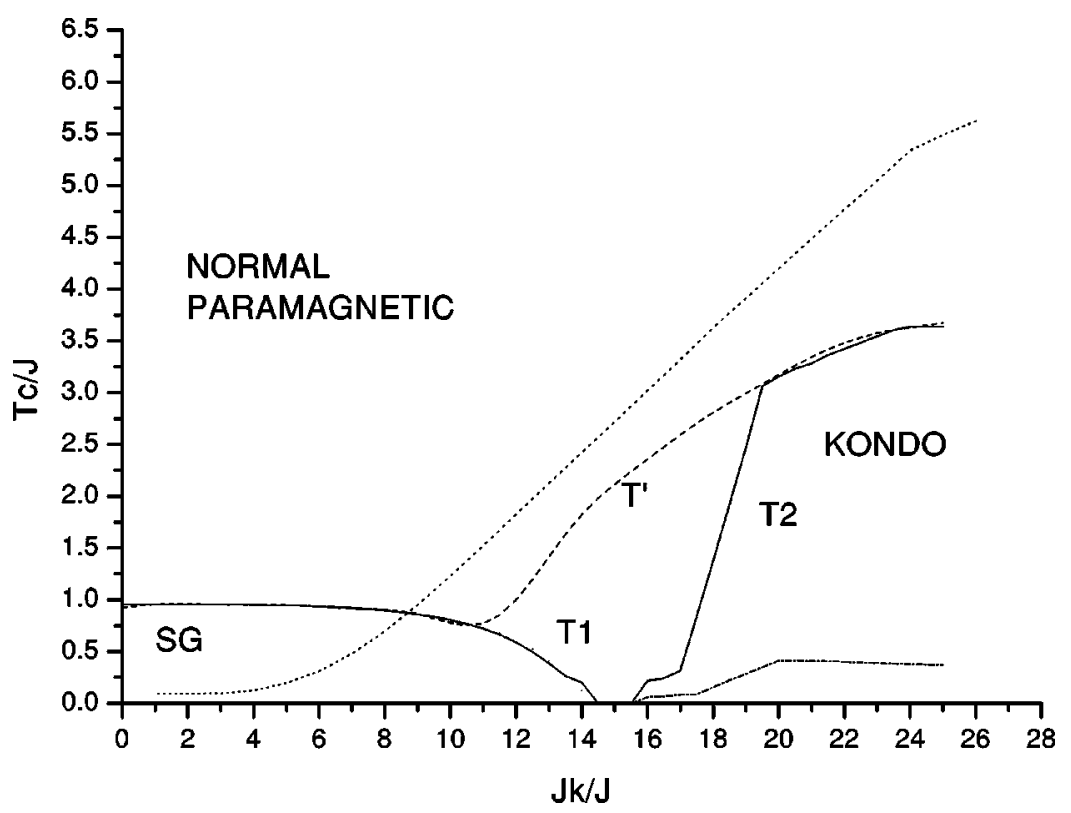

FIG. 1. Phase diagram in the $T-J_{K}$ plane as a function of $T / J$ and $J_{K} / J$ for the transverse field $\Gamma=\alpha J_{K}^{2}, \alpha J=0.01348 \quad$ (solid line) and $\alpha J$ $=0.01344$ (dashed line), for the conduction bandwidth $D / J=12$. The critical (solid) second order line $T_{1}$ for low values of $J_{K}$ separates the paramagnetic phase $(q=\lambda=0)$ for high temperatures from the spin glass phase $(q \geqslant 0, \lambda=0)$ at low temperatures and ends at a QCP. The critical second order (solid) line $T_{2}$ for large values of $J_{K}$ separates the paramagnetic phase from the Kondo phase $(\lambda \geqslant 0, q=0)$ and ends at a second QCP. The critical (dashed) line $T^{\prime}$ does not have a QCP. The dash-dotted line represents the "pure" Kondo temperature $T_{K}$ and the dotted line represents the residual magnetic moment $\bar{\chi}_{c}\left(J_{K}\right)$ on the line $T=T_{2}$

where we considered $D / J=12$ and two values of $\alpha J$. The two cases corresponding to these two values of $\alpha J$ are given in Fig. 1. The first case (represented by full lines) corresponds to the really interesting situation of the phase diagram with two QCPs, while the second case (represented by the dashed line $T^{\prime}$ ) is plotted here for comparison to show the case without a QCP.

In the first case, corresponding to $\alpha J=0.01348$ (solid line) the second order critical line $T_{1}\left(J_{K}\right)$ that separates the paramagnetic from the spin glass phase ends at a QCP corresponding to a $J_{K}$ value called $J_{K 1}^{c}$ here, while the second order critical line $T_{2}\left(J_{K}\right)$ that separates the paramagnetic from the Kondo phase ends at a second QCP $J_{K 2}^{c}$ and we have here $J_{K 2}^{c}>J_{K 1}^{c}$.

An asymptotic calculation at low temperatures gives

$$
k_{B} T_{1}=\frac{\sqrt{2}}{3}\left[2 \sqrt{2} J-\alpha J_{K}^{2}\right]
$$

and the corresponding value for $J_{K 1}^{c}$ at the QCP is given by $\alpha\left(J_{K 1}^{c}\right)^{2}=2 \sqrt{2} J$. On the other hand, close to the second QCP an asymptotic calculation gives

$$
k_{B} T_{2} \approx \log \left(T_{K}^{-1} \frac{\alpha\left(J_{K}\right)^{2}}{1+\alpha\left(J_{K}\right)^{2} / D}\right),
$$

where $T_{K}=D \exp \left(-2 D / J_{K}\right)$. Thus the value of $J_{K 2}^{c}$ is the solution of $T_{K}\left(J_{K 2}^{c}\right)=\alpha\left(J_{K 2}^{c}\right)^{2}$ in the asymptotic limit of very large $D$ values. In order to obtain a solution $J_{K 2}^{c}$ that makes $T_{2}=0$ in Eq. (39) it is necessary that $4 \alpha D=0.6473$, what gives $\alpha J=0.013485$ for $D=12 J$, and for these values of the parameters in Fig. 1, we have obtained two QCPs with $J_{K 2}^{c}>J_{K 1}^{c}$. In the second case corresponding to a smaller value of $\alpha, \alpha J=0.01344$, the two QCPs disappear and we obtain a single transition line (dashed line $T^{\prime}$ ) separating the paramagnetic from the ordered (spin glass and Kondo) phases. The physical case shown in Fig. 1, where the two QCPs are very close to each other and almost equal, corre- sponds well to experimental phase diagrams obtained in some cerium or uranium disordered alloys. We obtain from Eq. (34) in the disordered region that $\bar{\chi} \rightarrow 0.5$ when $\beta \rightarrow 0$, thus giving the expected Curie-Weiss behavior for the static susceptibility $\chi=\beta \bar{\chi}$, while for $\beta \rightarrow \infty$ we get $\chi \rightarrow \Gamma$ $+\sqrt{\Gamma^{2}-8 J^{2}}$ when $\Gamma=\alpha J_{K}^{2}>2 \sqrt{2} J$. We recover here the square root singularity in the linear static susceptibility that is present in other quantum spin glass models. ${ }^{20-22}$ It is interesting to remark that $\bar{\chi}=\chi T=S(S+1)$ is the residual effective localized spin in Curie-Weiss theory. The value for $\bar{\chi}$ on the critical line $T_{2}\left(J_{K}\right)$ is presented in Fig. 1, where we notice the steep drop to almost zero close to the QCP $J_{K 2}^{c}$.

\section{CONCLUSIONS}

We study in this paper the phase transitions in a heavy fermion system represented by a Hamiltonian that couples the $S_{z}$ components of the localized spins of a Kondo lattice ${ }^{7}$ with random, long range interactions, like in the SK model for a spin glass, ${ }^{18}$ while the transverse components are acted upon by a field in the $x$ direction. ${ }^{24}$ As the transverse field $\Gamma$ mimics the spin flip part of the Heisenberg coupling among localized spins, that originates in the RKKY interaction, we assume that $\Gamma \approx \alpha J_{K}^{2}$ where $J_{K}$ is the antiferromagnetic Kondo coupling. ${ }^{6}$ Using functional integrals techniques and a static, replica symmetric Ansatz for the Kondo and spin glass order parameters, we derive a mean-field expression for the free energy and the saddle-point equations for the order parameters. The use of the static Ansatz in the case of the transverse spin glass is justified, because it is the singularity of the zero frequency mode that determines the critical line. ${ }^{20-22}$ The numerical solution of the saddle-point equations allows us to draw the magnetic phase diagram in the $J_{K}$ vs $T$ plane (presented in Fig. 1) for fixed values of $J$ and $D$ with $D / J=12$ and for two values of $\alpha J, \alpha J=0.01348$ or $\alpha J=0.01344$.

Figure 1 shows three distinct phases. At high temperatures, the "normal" phase is paramagnetic with vanishing 
Kondo and spin glass order parameters, i.e., $\lambda=q=0$. When temperature is lowered, for not too large values of the ratio $J_{K} / J$, a second-order transition line is found at $T=T_{1}$ to a spin glass phase with $q>0$ and $\lambda=0$. The critical line $T_{1}\left(J_{K}\right)$ ends at a quantum critical point $J_{K 1}^{c}$. Finally, for large values of the ratio $J_{K} / J \geqslant J_{K 2}^{c} / J$, where $J_{K 2}^{c} \geqslant J_{K 1}^{c}$, there is a second order transition line to a Kondo state with $\lambda \geqslant 0$. These results are very sensitive to the value of $D / J$ and $\alpha J$. For $D / J=12$ and $\alpha J \leqslant 0.01344$ the QCP disappear while they are favored for larger values of $\alpha J$. We can also remark that we get here only "pure" Kondo or SG phases and never a mixed SG-Kondo phase with the two order parameters different from zero as already noticed for the competition between Kondo and antiferromagnetic phases; ${ }^{28,29}$ this result is probably connected to the approximations used here to treat the starting Hamiltonian. A QCP has been observed experimentally in several cerium and uranium disordered alloys $^{12-14}$ and the phase diagram shown in Fig. 1 which yields two QCPs improves considerably the description of the spin glass-Kondo transition with respect to previous publications. ${ }^{15-17}$ However, the experimental situation is generally not really clear; for example, there is no experimental information on the precise nature of the SG-Kondo transition in $\mathrm{CeNi}_{1-x} \mathrm{Cu}_{x}$ alloys. ${ }^{10}$ Moreover, the phase diagrams of several systems involve also an antiferromagnetic phase and this case is theoretically studied elsewhere. ${ }^{17}$ Our theoretical results describe also the spin glass and Kondo phases in uranium alloys. An unsolved basic question concerns also the existence or not of a "mixed" SG-Kondo phase in cerium and uranium disordered alloys and this problem is worth being studied experimentally in more detail. Thus further experimental work is necessary, but our model yields a new striking point in the behavior of heavy fermion disordered alloys in the vicinity of the quantum critical point.

\section{ACKNOWLEDGMENTS}

We acknowledge enlightening discussions with J. C. Gómez Sal. This work is partially supported by Conselho Nacional de Pesquisa e Desenvolvimento (CNPQ) and by Financiadora de Estudos e Projetos (FINEP). One of us (B.C.) would like to thank the French-Brazilian CNRSCNPq cooperation.
${ }^{1}$ S. Doniach, Physica B \& C 91, 231 (1977).

${ }^{2}$ B. Barbara, H. Bartholin, D. Florence, M. F. Rossignol, and E. Walker, Physica B \& C 86-88, 177 (1977).

${ }^{3}$ T. Graf, J. D. Thompson, M. F. Hundley, R. Movshovich, Z. Fisk, D. Mandrus, R. A. Fischer, and N. E. Phillips, Phys. Rev. Lett. 78, 3769 (1997).

${ }^{4}$ K. Alami-Yadri, H. Wilhelm, and D. Jaccard, Physica B 259-261, 157 (1999).

${ }^{5}$ G. Knebel, D. D. Braithwaite, G. Lapertot, P. C. Canfield, and J. Flouquet, J. Phys.: Condens. Matter 13, 10935 (2001).

${ }^{6}$ J. R. Iglesias, C. Lacroix, and B. Coqblin, Phys. Rev. B 56, 11820 (1997).

${ }^{7}$ B. Coqblin, C. Lacroix, M. A. Gusmão, and J. R. Iglesias, Phys. Rev. B 67, 064417 (2003).

${ }^{8}$ S. R. Julian, C. Pfleiderer, F. M. Grösche, N. D. Mathur, G. J. MacMullan, A. J. Divier, I. R. Walker, and G. G. Lonzarich, J. Phys.: Condens. Matter 8, 9675 (1996); H. von Löhneysen, ibid. 8, 9686 (1996).

${ }^{9}$ P. Coleman, Physica B 259-261, 353 (1999).

${ }^{10}$ J. Garcia Soldevilla, J. C. Gomez Sal, J. A. Blanco, J. I. Espeso, and J. Rodriguez Fernandez, Phys. Rev. B 61, 6821 (2000).

${ }^{11}$ J. C. Gómez Sal, J. Garcia Soldevilla, J. A. Blanco, J. I. Espeso, J. Rodriguez Fernandez, F. Luis, F. Bartolomé, and J. Bartolomé, Phys. Rev. B 56, 11741 (1997).

${ }^{12}$ S. Majumdar, E. U. Sampathkumarar, St. Berger, M. Della Mea, H. Mickor, M. Brando, J. Hemberger, and A. Loidl, Solid State Commun. 121, 665 (2002).

${ }^{13}$ R. Vollmer, T. Pietrus, H. V. Löneyssen, R. Chau, and M. B. Maple, Phys. Rev. B 61, 1218 (2000).

${ }^{14}$ V. S. Zapf, R. P. Dickey, E. J. Freeman, C. Sirvent, and M. B.
Maple, Phys. Rev. B 65, 024437 (2001).

${ }^{15}$ A. Theumann, B. Coqblin, S. G. Magalhães, and A. A. Schmidt, Phys. Rev. B 63, 054409 (2001).

${ }^{16}$ S. G. Magalhães, A. A. Schmidt, A. Theumann, and B. Coqblin, Eur. Phys. J. B 30, 419 (2002).

${ }^{17}$ S. G. Magalhães, A. A. Schmidt, F. M. Zimmer, A. Theumann, and B. Coqblin, Eur. Phys. J. B 34, 447 (2003).

18 D. Sherrington and S. Kirkpatrick, Phys. Rev. Lett. 35, 1792 (1975); S. Kirkpatrick and D. Sherrington, Phys. Rev. B 17, 4384 (1978).

${ }^{19}$ A. Georges, O. Parcollet, and S. Sachdev, Phys. Rev. B 63, 134406 (2001).

${ }^{20}$ J. Ye, S. Sachdev, and N. Read, Phys. Rev. Lett. 70, 4011 (1993).

${ }^{21}$ N. Read, S. Sachdev, and J. Ye, Phys. Rev. B 52, 384 (1995).

${ }^{22}$ J. Miller and D. A. Huse, Phys. Rev. Lett. 70, 3147 (1993).

${ }^{23}$ Y. Goldschmidt and P.-L. Lai, Phys. Rev. Lett. 64, 2467 (1990), and references therein.

${ }^{24}$ A. Theumann, A. A. Schmidt, and S. G. Magalhães, Physica A 311, 498 (2002).

25 J. W. Negele and H. Orland, Quantum Many Particles (AddisonWesley, Reading, MA, 1987); V. N. Popov, Functional Integrals and Collective Excitations (Cambridge University Press, Cambridge, England, 1987).

${ }^{26}$ C. Lacroix and M. Cyrot, Phys. Rev. B 20, 1969 (1979).

${ }^{27}$ A. M. Sengupta and A. Georges, Phys. Rev. B 52, 10295 (1995).

${ }^{28}$ B. Coqblin, M. A. Gusmão, J. R. Iglesias, C. Lacroix, A. Ruppenthal, and Acirete S. da Rosa Simões, Physica B 281-282, 50 (2000).

${ }^{29}$ H. Tsunetsugu, M. Sigriot, and K. Ueda, Rev. Mod. Phys. 69, 809 (1977). 